\title{
Coherence-length dependence of fluctuations in the conductance of normal-superconducting interfaces
}

\author{
J. Bruun \\ School of Physics and Materials, Lancaster University, Lancaster LA1 4YB, United Kingdom \\ V. C. Hui \\ D.R.A., Electronics Division, Malvern, WR1 3PS, United Kingdom \\ C. J. Lambert \\ School of Physics and Materials, Lancaster University, Lancaster LA1 4YB, United Kingdom
}

(Received 30 September 1993)

\begin{abstract}
We compute sample-to-sample fluctuations in the electrical conductance $G_{N S}$ of mesoscopic normalsuperconducting boundaries. If the superconductor is clean and the normal conductor diffusive, the rms deviation $\delta G_{N S}$ is approximately a factor of 2 larger than the universal value obtained for normal disordered conductors. This is consistent with predictions of random matrix theories and recent numerical simulations. If the normal material is clean, but the superconductor diffusive, the boundary conductance fluctuation is greater than $\delta G_{N S}$, by an amount which increases as the superconducting coherence length decreases. For a diffusive normal material in contact with a dirty superconductor, $\delta G_{N S}$ is insensitive to weak disorder in the superconductor, but decreases in the extreme dirty limit, where quasiparticles within the superconductor become Anderson localized.
\end{abstract}

\section{INTRODUCTION}

When a scattering region is smaller than the quasiparticle phase-coherence length $l_{\phi}$, an excitation undergoes only elastic scattering and transport properties are describable through the single-particle Schrödinger equation, or in the presence of superconductivity, through the Bogoliubov-de Gennes equation. In the absence of superconductivity, mesoscopic structures of this kind have been realized experimentally for many years and the associated transport theory has reached a high level of maturity. ${ }^{1,2}$ In the presence of superconductivity, the field is much less well developed. Recent experiments on the electrical conductance of normal-superconducting interfaces $^{3-6}$ and normal-superconducting loops ${ }^{7}$ have demonstrated that coherent transport in the presence of superconductivity can now be observed and a several theoretical treatments ${ }^{8-16}$ have begun to incorporate superconductivity into the transport theory of normal mesoscopic systems.

One theoretical development of particular interest is the prediction of universal fluctuations in the boundary conductance of a normal-superconducting interface. In normal, diffusive mesoscopic samples, it is known ${ }^{17-19}$ that if the microscopic impurity configuration of a given sample is perturbed, the change in the electrical conductance $G$ is of the order $e^{2} / h$ and is independent of the $G$. More precisely for an ensemble of samples with a given shape, the rms deviation $\delta G=A e 2 / h$, where the prefactor $A$ is independent of the ensemble averaged conductance $\langle G\rangle$ and depends only on the geometry of the samples.

Recently Takane and Ebisawa ${ }^{13,14}$ and Beenakker and co-workers ${ }^{15,16}$ have demonstrated that universal conductance fluctuations at normal-superconducting interfaces are enhanced by a factor of order 2 , compared with corresponding normal systems. To simplify the analysis, the work of Refs. 13-16 was restricted to systems in which a disordered normal $(N)$ region is connected to a clean superconductor $(S)$ with a uniform superconducting order parameter. In these calculations the normal scattering region is mesoscopic and the disorder is physically separate from the superconductor. Consequently, when the superconducting coherence length is orders of magnitude greater than the Fermi wavelength, a quasiparticle transmitted through the $(N)$ region and impinging on the superconductor, is Andreev reflected with probability unity.

In this paper we extend the work of Refs. $13-16$ by allowing the disorder to exist in both the normal and superconducting regions. By relaxing this restriction, we are able to investigate whether or not universal conductance fluctuations survive in the presence of more realistic interfaces and to address questions concerning the dependence of the size of the fluctuations on the superconducting coherence length. For a disordered normal region in contact with a clean superconductor, our results agree with those of Refs. 13-16. For a clean, normal region in contact with a dirty superconductor, we demonstrate that conductance fluctuations remain universal, in the sense that provided the disorder is weak, the magnitude of the fluctuation is independent of the size of the boundary conductance, but with a value which increases as the superconducting coherence length is decreased.

\section{FORMULAS FOR THE ELECTRICAL CONDUCTANCE OF MESOSCOPIC SUPERCONDUCTORS}

In this section we highlight relevant formulas needed to calculate the electrical conductance of phase-coherent 
normal and superconducting heterostructures and briefly mention how these can be evaluated using a numerical technique, outlined in Ref. 12 and introduced earlier to investigate Anderson localization in disordered superconductors. ${ }^{20-22}$

During the past decade, two exact formulas have been derived for the conductance of a mesoscopic normalsuperconducting structures. First the Blonder-TinkhamKlapwijk (BTK) formula was introduced to describe scattering at a normal-superconducting interface ${ }^{8}$ and later the Lambert formula was derived for an arbitrary superconducting region attached by normal leads to external reservoirs. ${ }^{9}$ In Ref. 9 both the well-known Landauer formula and the two-probe Büttiker formula were generalized to include Andreev scattering. During the past two years, both the above formulas have been rederived $^{10}$ and the work of Ref. 9 extended to multiprobe structures. ${ }^{11,12}$ With the additional assumption that a quasiparticle entering a clean superconducting lead is Andreev reflected with probability one, Beenakker has also rewritten the BTK result in a form more convenient for applying random matrix techniques. ${ }^{15}$ Since the analysis of Refs. 13 and 14 is in broad agreement with Refs. 15 and 16, the latter assumption would appear to be justified, provided the superconductor is clean and the superconducting coherent length is several orders of magnitude greater than the Fermi wavelength.

In what follows, to avoid restrictions on the nature of the disorder, all results are obtained by evaluating the zero-temperature Lambert formula, ${ }^{9,11,12}$ which yields for the two-probe electrical conductance $G$ in units of $2 e^{2} / h$,

$$
G=T_{0}+T_{a}+\frac{2\left(R_{a} R_{a}^{\prime}-T_{a} T_{a}^{\prime}\right)}{R_{a}+R_{a}^{\prime}+T_{a}+T_{a}^{\prime}}
$$

The coefficients $R_{0}, T_{0}\left(R_{a}, T_{a}\right)$ are probabilities for normal (Andreev) reflection and transmission for zero-energy quasiparticles from one reservoir, while $R_{0}^{\prime}, T_{0}^{\prime}\left(R_{a}^{\prime}, T_{a}^{\prime}\right)$ are corresponding probabilities for quasiparticles from the second reservoir. These satisfy $T_{0}+T_{a}=T_{0}^{\prime}+T_{a}^{\prime}$ and

$$
R_{0}+T_{0}+R_{a}+T_{a}=R_{0}^{\prime}+T_{0}^{\prime}+R_{a}^{\prime}+T_{a}^{\prime}=N_{0},
$$

where $N_{0}$ is the number of zero-energy quasiparticle channels in the leads. In general the conductance $G$ cannot be regarded as arising from boundary scattering, except in the limit of zero transmission, when Eq. (1) simplifies to

$$
G^{-1}=\left(G_{N S}\right)^{-1}+\left(G_{N S}^{\prime}\right)^{-1}=\left(2 R_{a}\right)^{-1}+\left(2 R_{a}^{\prime}\right)^{-1} \text {. }
$$

The quantities $G_{N S}=2 R_{a}$ and $G_{N S}^{\prime}=2 R_{a}^{\prime}$ are simply BTK boundary conductances and therefore in this limit, the left and right boundary resistances with the external leads simply add in series.

As discussed in detail elsewhere $e^{9,11,12}$ all coefficients in Eqs. (1) and (2) are obtained by summing the squared modulii of elements of submatrices of the multichannel scattering matrix $S$ of the system. For systems described by a tight-binding Hamiltonian, ${ }^{12,22}$ the latter can be obtained by employing a transfer-matrix technique to solve the Bogoliubov-de Gennes equation,

$$
H\left[\begin{array}{l}
\psi(\underline{r}) \\
\phi(\underline{r})
\end{array}\right]=E\left[\begin{array}{l}
\psi(\underline{r}) \\
\phi(\underline{r})
\end{array}\right],
$$

where

$$
H=\left(\begin{array}{cc}
H_{0} & \Delta \\
\Delta^{*} & -H_{0}^{*}
\end{array}\right) .
$$

In what follows, $H_{0}$ is chosen to be a tight-binding Anderson model on a square lattice, with diagonal disorder. Thus $\left(H_{0}\right)_{i j}=\epsilon_{j} \delta_{i j}-v \delta_{j j_{i}^{\prime}}$, where $j_{i}^{\prime}$ is a nearest neighbor of $i$, with a nearest-neighbor hopping element $v$. The diagonal elements, $\epsilon_{j}$ are random numbers uniformly distributed between $\epsilon_{0} \pm \frac{1}{2} W_{N}$ and $\epsilon_{0} \pm \frac{1}{2} W_{S}$ in the normal and superconducting materials, respectively. In Eq. (3), $\Delta$ is a diagonal order-parameter matrix, with elements $\Delta_{i j}=\Delta_{j} \delta_{i j}$. For a site $j$ in the superconductor, $\Delta_{j}=\Delta_{0}$, otherwise $\Delta_{j}=0$. For sites $i$ belonging to the external leads, $\Delta_{i}=\epsilon_{i}=0$.

A similar tight-binding approach has been adopted in Ref. 14. The key difference here is that the restriction to clean superconductors will not be imposed. In what follows we consider the two-dimensional sample, depicted in Fig. 1. The device is $M$ sites wide and $L=L_{N}+L_{S}$ sites long, with normal and superconducting regions of length $L_{N}, L_{S}$, respectively. In the normal limit, where all Andreev terms vanish, Eq. (1) reduces to the well-known Büttiker formula, ${ }^{23} G=T_{0}$. In this limit, $L_{S}=0$ and in the Ohmic regime for fixed normal disorder $W_{N}$, one can determine the elastic mean free path $l$ by varying $L$ and using the relation $\langle G\rangle \sim(M / L) l$, where the angular brackets denote an average over all impurity configurations. Once the mean free path is known, the size $L_{N}$ of the normal region can be chosen to render it ballistic $\left(L_{N}<l\right)$ or diffusive $\left(L_{N} \gtrsim l\right)$.

In what follows all calculations are performed with energy $E=0$, a hopping strength $v=1$ and periodic boundary conditions in the transverse direction. The rms fluctuations $\delta G=\sqrt{\left\langle\left(G^{2}-\langle G\rangle^{2}\right)\right\rangle}$ are evaluated from 2000 realizations of the disordered Hamiltonian $H_{0}$. It should be noted that the number of open channels in the normal leads is a discontinuous function of $E$; a feature which leads to observable conductance steps in thin quantum wires. For $M$ even, such a step occurs at $E=\epsilon_{0}$ and there-

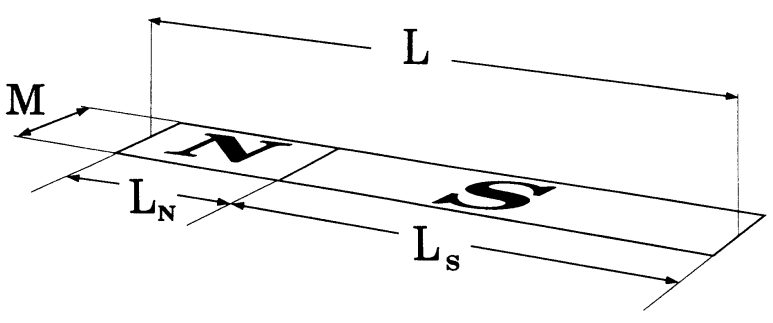

FIG. 1. The normal-superconducting scattering region. The geometry is quasi-one-dimensional with width $M \ll L$, where $L=L_{N}+L_{S}$. Uniform disorder can be present in the normal segment $W_{N}$ and the superconductor $W_{S}$. 
fore to avoid a discontinuity, a site energy in the leads of $\epsilon_{0}=10^{-6}$ was chosen.

\section{RESULTS}

To make contact with earlier numerical work, ${ }^{14}$ we first examine the elastic mean free path $l$ for a purely normal conductor. For this purpose we plot $\langle G\rangle_{L / M}$ versus $L$ for three different widths, $M=4,8$, and 16, corresponding to $N_{0}=3,7$, and 15 open channels, respectively. Examples of such plots, obtained with a fixed disorder of $W_{N}=1$, are shown in Fig. 2. In the Ohmic regime, the quantity $\langle G\rangle L / M$ should be independent of $L$. However, for finite width systems, such behavior persists for only a limited range of $L$, because for small $L$ the sample is ballistic and for large $L$, the system exhibits localized behavior, characteristic of quasi-one-dimensional structures. In Fig. 2 only the widest system $M=16 \mathrm{ex}-$ hibits a clear Ohmic plateau. For smaller widths, we follow Ref. 14 and heuristically associate $l$ the extremal value of $\langle G\rangle L / M$, to yield $l \sim 7,12$, and 14 for $M=4,8$, and 16, respectively. Using the above technique, the mean free path of a normal region, in the absence of superconductivity, may be estimated for any system size and disorder.

Consider now the system shown in Fig. 1, with a normal region of size $L_{N}$, mean free path $l$, connected to a superconducting region of size $L_{S}$. To the left of the normal region and to the right of the superconductor are a perfect normal leads (not shown), which carry quasiparticles to external reservoirs. In the limit that $L_{s} \gg \xi_{s}$, where $\xi_{s}$ is the superconducting coherence length, or equivalently in the limit $L_{s} \gg 1 / \Delta_{0}$, all transmission coefficients are negligibly small and Eq. (2) can be employed. Consequently by computing the scattering matrix $S$ of the system shown in Fig. 1, we can simultaneously obtain the conductance $G_{N S}$ of the left interface between a dirty normal material and the superconductor and the conductance $G_{N S}^{\prime}$ of the right-hand interface between the superconductor and a clean normal lead.

With this NS device we first investigate the situation in

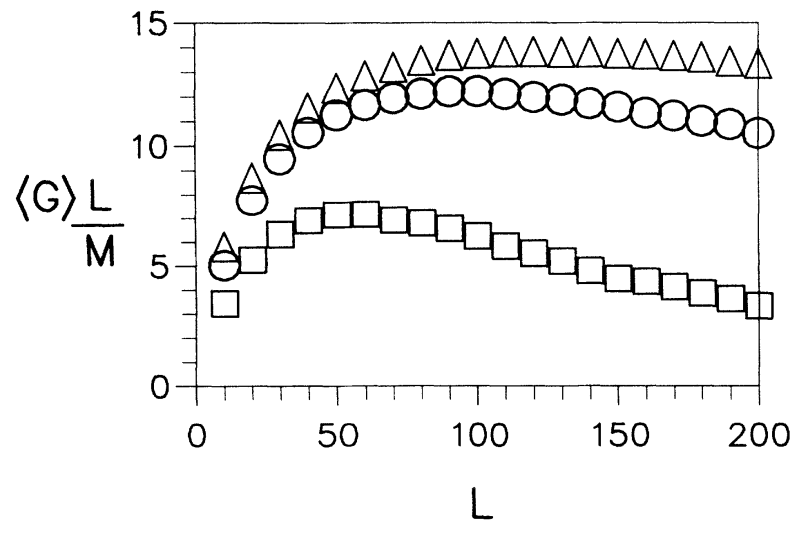

FIG. 2. For a fixed normal disorder of $W_{N}=1$, this shows plots of $\langle G\rangle L / M$ as functions of the system length $L$ for three widths, $M=4(\square), 8(\bigcirc)$, and $16(\triangle)$. which there is no disorder in the superconductor. For a system of width $M=8$, a normal region of length $L_{N}=120$ and various normal disorders $W_{N}$, ranging from 0 to 1, Fig. 3 shows results for both the average left boundary conductance $G_{N S}$ (lower figure) and the fluctuations $\delta G_{N S}$ (upper figure), plotted as functions of $L_{N} / l$, where $l$ is the mean free path of the normal region. In these calculations, $\Delta_{0}=0.1, L_{s}=400$, and quasiparticle transmission is negligible. For comparison the average conductance $G_{N}$ and fluctuations $\delta G_{N}$ in the absence of the superconductor are also shown.

Figure 3 shows that whereas both $G_{N}$ and $G_{N S}$ change significantly as the normal disorder (or equivalently $l$ ) is varied, the fluctuations are almost constant, reflecting universality. Furthermore $\delta G_{N S} / \delta G_{N}$ is of order 2, in qualitative agreement with Refs. 13-16. The fluctuations do in fact vary slightly with $W_{N}$, due to the finite width of the system. For $L_{N} / l=10$ (i.e., $W_{N}=1$ ) we find $\delta G_{N S} / \delta G_{N} \simeq 1.8$, with $\delta G_{N} \simeq 0.33$ and for $L_{N} / l=5$ (i.e, $W_{N}=0.6$ ) we find $\delta G_{N S} / \delta G_{N} \simeq 2$, with $\delta G_{N} \simeq 0.36$. The latter result compares favorably with a value $\delta G_{N} \simeq 0.37$ quoted by Lee and Stone. ${ }^{19}$

Having examined the interface between a dirty normal region and a clean superconductor, we now consider the situation in which the disorder $W_{S}$ within the superconductor becomes nonzero. In what follows, we fix the normal disorder at $W_{N}=0.6$, corresponding to $L_{N} / l=5$ and show results for a system of size $M=8, L_{N}=120$, $L_{S}=400$, with $\Delta_{0}=0.1$. Figure 4 (a) shows the left and right boundary conductances $G_{N S}, G_{N S}^{\prime}$ and their fluctuations $\delta G_{N S}, \delta G_{N S}^{\prime}$, as a function of the disorder $W_{S}$ in the superconductor. Figure 4(a) shows that for moderate values of $W_{S}, G_{N S}$ and $\delta G_{N S}$ are independent of $W_{S}$. In contrast the right-hand boundary conductance $G_{N S}^{\prime}$ decreases monotonically with increasing $W_{S}$, while the fluctuations $\delta G_{N S}^{\prime}$ rise to a plateau and thereafter remain

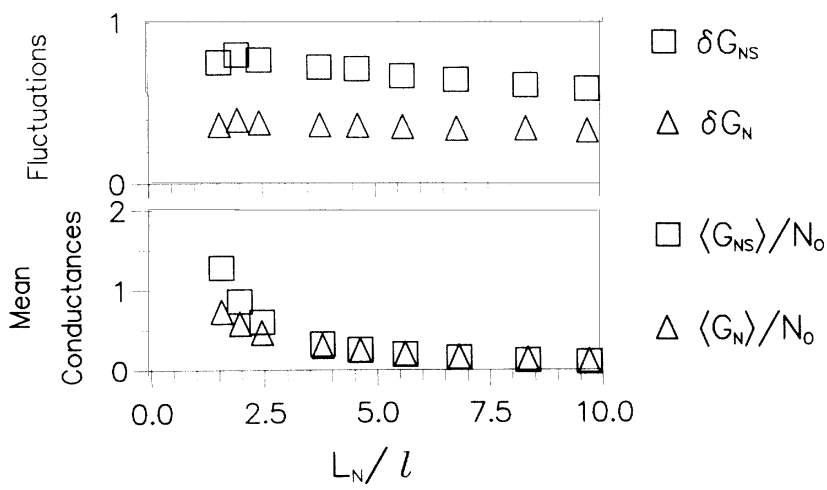

FIG. 3. In the upper figure, the ( $\triangle$ )'s show results for $\delta G_{N}$ and the ( $\square$ )'s show results for $\delta G_{N S}$ as functions of the ratio $L_{N} / l$, obtained by varying the normal disorder $W_{N}$ from 1 to 0 . The lower figure shows corresponding results for the average conductances per channel $\left\langle G_{N}\right\rangle / N_{0}(\triangle)$ and $\left\langle G_{N S}\right\rangle / N_{0}(\square)$. These results were obtained for a system of width $M=8$, a normal region of length $L_{N}=120$, a clean superconducting region of length $L_{S}=400$ and an order parameter of magnitude $\Delta_{0}=0.1$. 


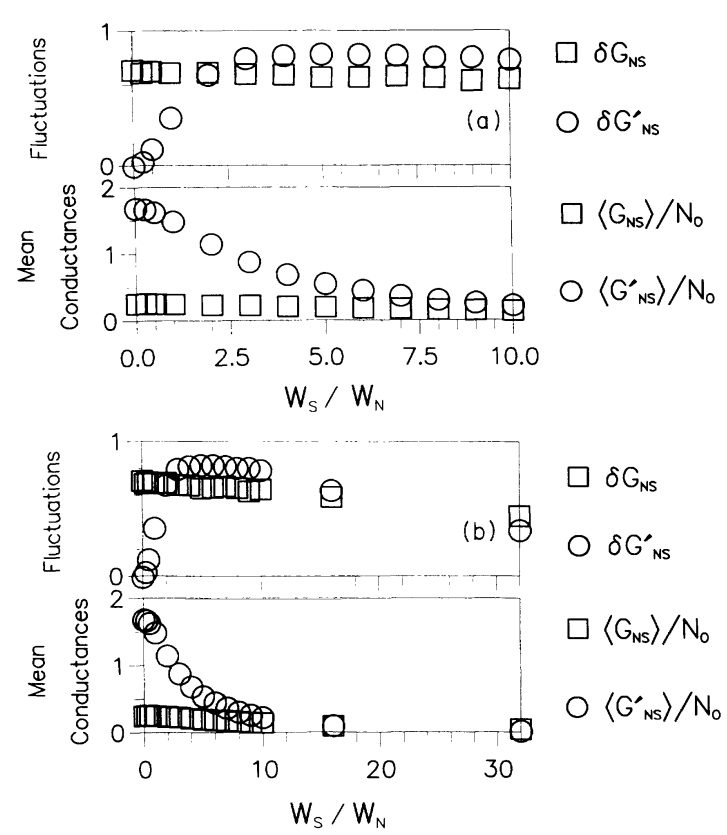

FIG. 4. For a system of width $M=8$, a normal region of length $L_{N}=120$, a superconducting region of length $L_{S}=400$ and an order parameter of magnitude $\Delta_{0}=0.1$, these graphs show results for the left and right boundary conductances and the associated fluctuations as functions of the disorder in the superconductor $W_{S}$. The disorder in the normal region is fixed at $W_{N}=0.6$. The values for $G_{N S}^{\prime}$ are (O) and for $G_{N S}$ they are ( $\square$ ). In (a) the disorder is varied from $W_{S}=0$ to $W_{S}=10 W_{N}$. (b) Extends the results of (a) to larger values of $W_{S}$, where strong elastic scattering dominates the transport.

constant over a finite range of disorder. Thus the conductance fluctuations associated with the boundary between a clean normal material and a dirty superconductor exhibit universality. However, it should be noted that the plateau value of $\delta G_{N S}^{\prime} \simeq 0.81$ is slightly greater than the left-hand value of $\delta G_{N S} \simeq 0.71$. In addition, as shown in Fig. 4(b), for large enough $W_{S}$, the fluctuations are suppressed.

This behavior can be understood qualitatively, by considering the superconducting region of width $M$, length $\xi_{s}=1 / \Delta_{0}$ at each of the interfaces. In the absence of disorder, quasiparticles penetrate a distance of order $\xi_{s}$ into the superconductor before Andreev reflecting and therefore one should ask whether or not a region of this extent is diffusive. For the moderate values of $W_{S}$ under in Fig. 4(a) this is indeed the case and therefore universal conductance fluctuations are observed. However, for the much larger values shown in Fig. 4(b) quasiparticles become Anderson localized ${ }^{20,21}$ and are normally reflected on a length scale $\alpha^{-1}<<\xi_{s}$, where $\alpha$ is the inverse localization length. Thus the boundary conductances and their fluctuations are suppressed.

The existence of a superconducting boundary layer of width $\xi_{s}=1 / \Delta_{0}$, also allows one to understand the result shown in Fig. 4(a) that the universal value of $\delta G_{N S}^{\prime}$ is greater than $\delta G_{N S}$. For normal diffusive systems, it is known that the precise value of the universal conduc-

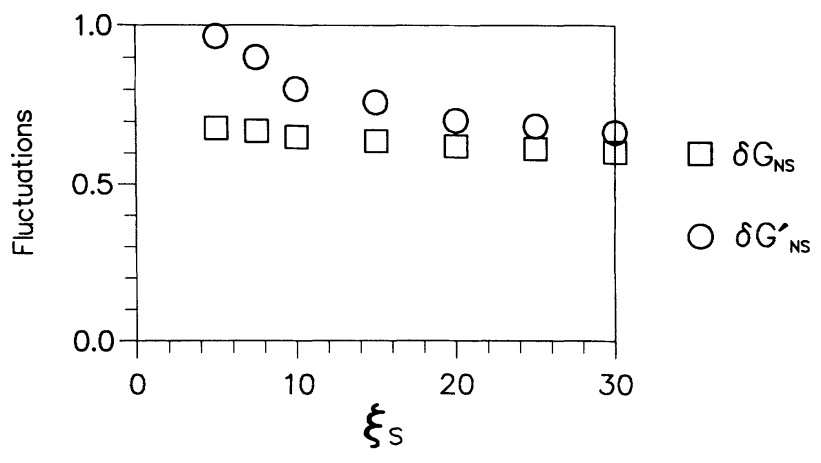

FIG. 5. For a sample of the same dimensions as Fig. 4 and with $W_{S}=8 W_{N}=4.8$, this figure shows how $\delta G_{N S}(\square)$ and $\delta G_{N S}^{\prime}$ (O) vary with the inverse order-parameter strength $\xi_{s}=1 / \Delta_{0}$.

tance fluctuations depends on the aspect ratio of a sample, varying from $\delta G_{N} \sim 0.37$ for quasi-one-dimensional samples, where $M / L \ll 1$, to $\delta G_{N} \sim 0.43$ when $M / L=1 .{ }^{19}$ When considering systems that incorporate superconductivity, the effective length of the conductor is of order $\xi_{s}$ and for this reason, in the limit that $\xi_{s} \gg M$, one expects the value of $\delta G_{N S}^{\prime}$ to decrease to the quasione-dimensional fluctuations characteristic of the lefthand $N S$ boundary to $\delta G_{N S}$. In Fig. 5 we show numerical results for the variation of $\delta G_{N S}$ and $\delta G_{N S}^{\prime}$ with the length scale $\xi_{s}$. The system used is identical to that used in Figs. 4, with the disorder within the superconductor fixed at $W_{S}=8 W_{N}$. From the length dependence of the normal conductance $G_{N}$, we estimate that the elastic mean free path is of order unity and the localization length is of order 50 . Figure 5 shows that $\delta G_{N S}$ is almost independent of the coherence length, whereas $\delta G_{N S}^{\prime}$ decreases monotonically with increasing $\xi_{s}$, approaching $\delta G_{N S}$ only in the limit $\xi_{s} / M \rightarrow \infty$.

\section{DISCUSSION}

In this paper, we have presented results for the interface conductance of a dirty superconductor in contact with both clean and dirty normal materials. Except in the limit of strong disorder, we have demonstrated that conductance fluctuations of the latter are unaffected by the disorder $W_{S}$ within the superconductor and are insensitive to the magnitude of the superconducting order parameter. In contrast for moderate values of $W_{S}$, the fluctuations associated with the interface between a dirty superconductor and a clean normal conductor, exhibit universal fluctuations, the precise value of which increases as $\Delta_{0}$ increases. We attribute this dependence to a change in the effective aspect ratio of the sample, experienced by quasiparticles entering the superconductor.

\section{ACKNOWLEDGMENTS}

This work was supported by the SERC, the European Community, NATO, and the MOD. It has benefited from useful conversations with S. J. Robinson, B. Kramer, J-L. Pichard, and J. H. Jefferson. 
${ }^{1}$ B. Kramer, Festkörperprobleme: Advanced Solid State Physics (Vieweg, Braunschweig, 1990), Vol. 30, p. 53.

${ }^{2}$ For a review of fluctuation phenomena in normal systems, see Mesoscopic Phenomena in Solids, edited by B. L. Al'tshuler, P. A. Lee, and R. A. Webb (North-Holland, Amsterdam,1991).

${ }^{3}$ A. Kastalsky, A. W. Kleinsasser, L. H. Greene, R. Bhat, F. P. Milliken, and J. P. Harbison, Phys. Rev. Lett. 67, 3026 (1991).

${ }^{4}$ Y. W. Kwong, K. Lin, P. J. Hakonen, M. S. Isaacson, and J. M. Parpia, Phys. Rev. B 44, 462 (1991).

${ }^{5}$ P. Santhanam, C. C. Chi, S. J. Wind, M. J. Brady, and J. J. Bucchignano, Phys. Rev. Lett. 66, 2254 (1991).

${ }^{6}$ C. Nguyen, H. Kroemer, and E. L. Hu, Phys. Rev. Lett. 69, 2847 (1992).

${ }^{7}$ V. T. Petrashov, V. N. Antonov, P. Delsing, and T. Claeson, Phys. Rev. Lett. 70, 347 (1993).

${ }^{8}$ G. E. Blonder, M. Tinkham, and T. M. Klapwijk, Phys. Rev. B 25, 4515 (1982).

${ }^{9}$ C. J. Lambert, J. Phys. Condens. Matter 3, 6579 (1991).

${ }^{10}$ Y. Takane and H. Ebisawa, J. Phys. Soc. Jpn. 61, 1685 (1992).
${ }^{11}$ C. J. Lambert, J. Phys. Condens. Matter 5, 707 (1993).

${ }^{12}$ C. J. Lambert, V. C. Hui, and S. J. Robinson, J. Phys. Condens. Matter 5, 4187 (1993).

${ }^{13}$ Y. Takane and H. Ebisawa, J. Phys. Soc. Jpn. 60, 3130 (1991).

${ }^{14}$ Y. Takane and H. Ebisawa, J. Phys. Soc. Jpn. 61, 2858 (1992).

${ }^{15}$ C. W. J. Beenakker, Phys. Rev. B 46, 12841 (1992).

${ }^{16}$ R. A. Jalabert, J-L. Pichard, and C. W. J. Beenakker, Phys. Rev. B 48, 2811 (1993).

${ }^{17}$ A. D. Stone, P. A. Mello, K. A. Muttalib, and J-L. Pichard, in Mesoscopic Phenomena in Solids, Ref. 2.

${ }^{18} \mathrm{~J}-\mathrm{L}$. Pichard, N. Zanon, Y. Imry, and A. D. Stone, J. Phys. (France) 51, 587 (1990).

${ }^{19}$ P. A. Lee and A. D. Stone, Phys. Rev. Lett. 55, 1622 (1985).

${ }^{20}$ C. J. Lambert and V. C. Hui, Physica B 165, 1107 (1990).

${ }^{21}$ V. C. Hui and C. J. Lambert, J. Phys. Condens. Matter 2, 7303 (1990).

${ }^{22}$ V. C. Hui and C. J. Lambert, J. Phys. Condens. Matter 5, 697 (1993).

${ }^{23}$ M. Büttiker, IBM J. Res. Dev. 32, 317 (1988). 